\title{
Clinical profile and risk stratification in multidrug-resistant tuberculosis (MDR-TB) and non-MDR-TB patients: A prospective study in a tertiary care hospital \\ Sunil Kumar $\mathbf{N}^{1, *}$, Priyashree $\mathbf{R}^{2}$, Venu Gopal $\mathbf{K}^{1}$ and Srikant Gadwalkar $\mathbf{R}^{1}$

\author{
${ }^{1}$ Department of General Medicine, Vijayanagara Institute of Medical Sciences, Bellary, Karnataka-583104, India \\ ${ }^{2}$ Department of General Medicine, Bangalore Medical College and Research Institute, Bangalore, Karnataka-560002, India
}

\begin{abstract}
Background: Multidrug resistant tuberculosis (MDR-TB) is a major threat for successful control of TB. Early diagnosis and drug sensitivity testing helps in improving disease prevalence. This study was undertaken to determine clinical profile and risk factors for MDR-TB and factors differentiating it from non MDR-TB.
\end{abstract}

Materials and methods: This is a prospective study conducted at a TB hospital attached to Vijayanagara institute of medical sciences, Bellary, Karnataka in South India over a period of 2 years. All patients diagnosed with MDRTB were studied for clinical parameters and another cohort of non MDR-TB in same study period were included for comparison. Clinical and radiological characteristics, and risk factors were compared between two groups.

Results: A total of 59 MDR-TB and 72 non MDR-TB patients were studied in this period. Males were predominant in both groups. Body mass index (BMI) was significantly low in MDR-TB group (18.5 vs $20.3 \mathrm{~kg} / \mathrm{m}^{2}$ ). Among clinical symptoms, breathlessness was significantly seen in MDR-TB group. Defaulter, failure and relapse were seen in $6.8 \%, 54.3 \%$ and $37.3 \%$ respectively. Cavitary lesions and reticulonodular patterns in imaging were significantly seen in MDR group. Eight patients in non MDR group had human immunodeficiency virus (HIV) infection compared to one patient in MDR.

Conclusion: Presence of cavity and reticulonodular patterns in imaging, significant breathlessness and lower BMI are significantly common in MDR TB patients. These parameters maybe considered for early suspicion and monitoring for drug resistance in index presentation.

Keywords: multidrug resistant TB; breathlessness; human immunodeficiency virus; cavity lesion; reticulonodular patterns

*Corresponding author: Dr. Sunil Kumar N, Senior Resident,
Department of General Medicine, Vijayanagara Institute of
Medical Sciences, Bellary, Karnataka-583104, India. Mobile No.:
+91 8971882160; Email: sunil.kumar.n6@gmail.com
Received 26 May 2021; Revised 15 July 2021; Accepted 22 July
2021; Published 30 August 2021
Citation: Kumar NS, Priyashree R, Gopal KV, Gadwalkar RS.
Clinical profile and risk stratification in multidrug-resistant tuberculosis (MDR-TB) and non-MDR-TB patients: A prospective study in a tertiary care hospital. J Med Sci Res. 2021; 9(4):199203. DOI: http://dx.doi.org/10.17727/JMSR.2021/9-30

Copyright: @ 2021 Kumar NS et al. Published by KIMS Foundation and Research Center. This is an open-access article distributed under the terms of the Creative Commons Attribution License, which permits unrestricted use, distribution, and reproduction in any medium, provided the original author and source are credited. 


\section{Introduction}

Multi drug resistant tuberculosis (MDR-TB) is defined as tuberculosis (TB) with resistance to at least rifampin and isoniazid. The treatment is longer and requires more toxic drugs than non MDR-TB $[1,2]$. Recurrence and treatment failure are also more common, with success rates measuring $48 \%$ globally [3]. Facilities for its diagnosis and treatment are limited in many high-burden countries like India $[1,4]$.

An useful incentive in this aspect is to predict those patients who are at risk of this challenging MDR-TB, so as to subject them for further testing and treating them in initial part of disease. Risk stratifying patients based on easily and rapidly-available clinical and/or laboratory data helps in vigorous monitoring of at risk patients. Several risk factors have been associated with MDR-TB. History of prior TB treatment, human immunodeficiency virus (HIV) infection, contact with a known MDR-TB patient, receipt of more than two treatment courses, lung cavitation, and bilateral lung disease [5-7]. Clinical characteristics, risk profile and prognosis may vary compared to non MDR-TB patients. There is scarcity in data understanding how MDR-TB patients differ in clinical profile as compared to patients with drug sensitive TB. Little is known about the relative contribution of clinical and other risk factors in high prevalence settings.

Hence, we undertook this prospective study in a community with high prevalence tuberculosis in northern part of Karnataka to assess clinical profile and risk factors associated with MDR-TB patients. Also, these parameters are compared with nonMDR-TB cohort to identify changes in characteristics as compared to MDR-TB patients.

\section{Materials and methods}

The study was conducted after the approval of institutional ethical committee. This is a prospective case-control study done in TB hospital attached to Vijayanagara institute of medical sciences, Bellary, Karnataka over a period of 2 years from January 2015 to December 2016. All Patients, aged more than 15 years of age who were presenting to TB hospital with symptoms of fever, cough, dyspnea, hemoptysis and diagnosed with MDR-TB were included in the study. Another cohort of patients who presented in this period with non MDR-TB are also studied. Clinical and demographic characteristics, patients duration of illness, co-morbidities, radiological findings were noted and these characteristics were compared with non MDR-TB cohort of patients. All patients were confirmed by positive sputum culture for Mycobacterium tuberculosis. Drug sensitivity testing (DST) against was also conducted for the Mycobacterium isolated. X-ray and contrast enhanced computed tomography (CT) scan of thorax was performed in all cases. Study participants were selected from both admitted as well as out-patients, including patients on active anti-TB treatment, those who died, defaulters of TB treatment, and those who had lost for follow up during the study period. Directly observed short therapy (DOTS) was implemented on all patients with non MDR-TB and DOTS-Plus for MDR-TB infections.

Patients not willing to be part of study, age less than 15 years and who were lost to follow up were excluded from study. Due to long duration of disease, patient's prognosis could not be ascertained and patients who died in the study period were also excluded.

\section{Statistics}

The data were analyzed descriptively using means, standard deviations and percentages. Tests of significance were performed using independent studentt-tests and chi-square analyses as appropriate for the variables used in the comparison. The level of significance was set at 0.05 . All the analyses were done with the statistical package for the social sciences for Windows version 16; SPSS, Chicago.

\section{Results}

A total of 59 patients got admitted in this period with confirmed MDR strain. Another 72 patients with nonMDR-TB were also included in the study. Males were predominantly involved in both the groups $(71.2 \%$ in MDR-TB group and 65.3\% in non-MDR-TB group). 30-44 years of age constituted $44.1 \%$ and $47.2 \%$ in MDR and non-MDR group respectively. Around $76 \%$ and $69 \%$ represented from village background in MDR and non-MDR group respectively. BMI was significantly low in MDR-TB group (18.5 vs 20.3 $\mathrm{kg} / \mathrm{m}^{2}$ ). Among clinical symptoms, fever, cough and weight loss were seen similarly in both the groups. Breathlessness was significantly seen in MDR-TB group (Table 1). 
Table 1: Comparison of clinical profile among MDR and non MDR-TB patients.

\begin{tabular}{|lccc|}
\hline Clinical profile & MDR & Non MDR & Pvalue \\
\hline Age(in years) & $32 \pm 15$ & $36 \pm 18$ & 0.25 \\
Gender (M:F) & $17: 42$ & $25: 47$ & 0.47 \\
BMI & $18.5 \pm 3.6$ & $20.3 \pm 3.1$ & 0.02 \\
Symptoms & & & \\
Cough & $43(72.9 \%)$ & $57(79.1 \%)$ & 0.40 \\
Fever & $24(40.7 \%)$ & $35(48.6 \%)$ & 0.36 \\
Breathlessness & $26(44.1 \%)$ & $18(25.0 \%)$ & 0.02 \\
Haemoptysis & $05(08.5 \%)$ & $04(05.6 \%)$ & 0.51 \\
Weight loss & $13(22.0 \%)$ & $14(19.4 \%)$ & 0.71 \\
\hline
\end{tabular}

Etiological profile of MDR-TB are depicted in table 2. Percentage of defaulter, failure and relapse were $6.8 \%, 54.3 \%$ and $37.3 \%$ respectively in MDR-TB cohort. Patients infected with HIV were one in MDR group and 8 in non-MDR group, which was significantly seen in non-MDR patients. Other comorbidities were similarly seen in both groups as described in table 2 .

Table 2: Description of MDR-TB characteristics and comorbidities in study population.

\begin{tabular}{|llll|}
\hline Characteristics & $M D R$ & Non MDR & P value \\
\hline H/o TB & $59(100 \%)$ & $18(25.0 \%)$ & 0.001 \\
H/o failure & $32(54.3 \%)$ & 00 & 0.001 \\
H/o defaulter & $04(06.8 \%)$ & 00 & 0.03 \\
H/o relapse & $22(37.3 \%)$ & 00 & 0.001 \\
First attack & 00 & $21(29.2 \%)$ & 0.001 \\
Contact history & $01(01.7 \%)$ & 00 & 0.26 \\
Diabetes mellitus & $04(06.8 \%)$ & $02(02.8 \%)$ & 0.27 \\
Smoking & $18(30.5 \%)$ & $28(38.9 \%)$ & 0.42 \\
Nicotine & $09(15.3 \%)$ & $06(08.3 \%)$ & 0.21 \\
consumption & $11(18.6 \%)$ & $15(20.8 \%)$ & 0.75 \\
Alcoholics & $01(01.7 \%)$ & $08(11.1 \%)$ & 0.03 \\
HIV & & & \\
\hline
\end{tabular}

Table 3 shows the radiological features among the study cohort. Cavitary lesions were significantly seen in MDR group (54.2\%) as compared to non-MDR (18\%). Reticulonodular patterns were seen in 33.3\% of non-MDR group which was statistically significant.
Pleural effusion was only seen in non MDR cohort (6 patients) which was also a significant differentiating radiological factor among the patients. Although fibrosis was seen predominantly in non MDR group (33.3\%), it was not statistically significant.

Table 3: Comparison of radiological findings among MDR and non MDR-TB patients

\begin{tabular}{|lccc|}
\hline $\begin{array}{l}\text { Radiological } \\
\text { features }\end{array}$ & MDR & Non MDR & P value \\
\hline Cavity & $32(54.2 \%)$ & $13(18.0 \%)$ & 0.001 \\
Collapse of lung & $07(11.9 \%)$ & $04(05.5 \%)$ & 0.22 \\
Fibrosis & $11(18.6 \%)$ & $24(33.3 \%)$ & 0.07 \\
Fluffy shadows & $08(13.5 \%)$ & $07(09.7 \%)$ & 0.58 \\
$\begin{array}{l}\text { Reticulonodular } \\
\text { patterns }\end{array}$ & $08(13.5 \%)$ & $24(33.3 \%)$ & 0.01 \\
Miliary pattern & $08(13.5 \%)$ & $09(12.5 \%)$ & 1.00 \\
Pleural effusion & 00 & $06(08.3 \%)$ & 0.03 \\
\hline
\end{tabular}

Hematological parameters are compared among two groups in table 4. Inflammatory markers ESR and CRP were significantly elevated in both the groups and were almost similar. Although albumin $(2.4 \pm 0.5$ $\mathrm{gm} / \mathrm{dl})$ ) and hemoglobin $(9.7 \pm 1.3 \mathrm{gm} / \mathrm{dl})$ were marginally lower in MDR group, it was statistically not significant. 16 patients died during this study period in MDR group and 6 patients died in non-MDR group. All these patients had respiratory failure as cause of death. These patients were excluded from the final statistics due to significantly severe disease which may confound other parameters.

Table 4: Comparison of haematological parameters among MDR and non-MDR-TB patients.

\begin{tabular}{|lccc|}
\hline Parameters & $\begin{array}{c}\text { MDR-TB } \\
\text { group }\end{array}$ & $\begin{array}{c}\text { Non MDR-TB } \\
\text { group }\end{array}$ & Pvalue \\
\hline $\begin{array}{l}\text { Hemoglobin } \\
\text { (gm/dl) }\end{array}$ & $9.7 \pm 1.3$ & $10.3 \pm 1.5$ & 0.81 \\
$\begin{array}{l}\text { WBC count } \\
\text { (cells/ microliter) }\end{array}$ & $8987 \pm 3211.5$ & $7865 \pm 5110.2$ & 0.67 \\
$\begin{array}{l}\text { Platelet count } \\
\text { (lakh cells/ }\end{array}$ & $2.1 \pm 0.9$ & $1.9 \pm 1.1$ & 0.88 \\
microliter) & & & \\
ESR & $65 \pm 21$ & $55 \pm 18$ & 0.65 \\
CRP & $40.2 \pm 18$ & $35.6 \pm 21$ & 0.99 \\
$\begin{array}{l}\text { Serum protein } \\
\text { (gm/dl) }\end{array}$ & $5.2 \pm 1.6$ & $5.9 \pm 2.1$ & 0.54 \\
$\begin{array}{l}\text { Albumin (gm/dl) } \\
\text { Creatinine (mg/ }\end{array}$ & $2.4 \pm 0.5$ & $2.9 \pm 0.4$ & 0.09 \\
dl) & $0.9 \pm 0.5$ & $1.1 \pm 0.6$ & 0.21 \\
\hline
\end{tabular}




\section{Discussion}

This study was undertaken to determine differences in baseline clinical characteristics, radiological parameters and co-morbidities between MDR and non MDR TB. MDR-TB cases have been increasing globally. Early detection and treatment are top priories to fight MDR. Identifying factors that determine high risk for MDR-TB is one of key step in this aspect.

In this study, patients in both groups were of younger age with mean age between 30-40 years. This shows that working group of patients are commonly affected and to monitor compliance to prevent failure or defaulter cases. Some of studies have shown that MDR-TB is significantly seen in younger population [8-10]. Male gender was predominant in both MDRTB and non-MDR-TB but no association was found between the two groups.

Several other studies, including a systematic review in Europe [12], have reported an association between HIV and MDR-TB. However our study showed that there is no significant risk of MDR in patients with HIV infection. This is similarly seen in many of endemic countries to both HIV and TB $[6,9$, 11]. Also, ART improves survival in MDR-TB patients infected with HIV. However drug interactions and side effects are more common in these patients. Diabetes was the major co-morbidity among MDR patients $[1,5]$. Whether diabetics are prone to MDR strain requires further studies with larger no. of population, but lower immunity are definitely prone for TB and secondary infections, which may increase morbidity and mortality. Among other risk factors, the use of illicit drugs, alcohol and smoking are associated with drug default $[13,14]$. However, it is possible that these high risk factors are a marker for other social and behavioral factors that render default more likely.

Among clinical symptoms, except for breathlessness, none of other symptoms showed differences among both groups. As expected, with more extensive and severe disease, breathlessness proportions were more in MDR patients. In one of Thailand study, hemoptysis was significantly seen in MDR group [9]. Low BMI was also significantly seen in MDR group. Several other studies have reported association between HIV, low initial body weight, co-morbidities/ co-infections and age with death from MDR TB [15,
16]. Among the radiological features, cavitary lesion and reticulonodular shadows were significantly seen among MDR group. These lesions may represent a source for chronic and resistant strains to develop. Collapse of lung and fluffy shadows were also predominant in MDR group.

Anemia, inflammatory markers and decreased albumin are commonly seen in both the groups and did not show statistical differences. Larson et al had shown higher CRP in patients with TB [17]. These inflammatory markers can also be used for monitoring response to ATT and a lack in decline should prompt underlying MDR status.Among MDR patients, $32 \%$ were because of failure, $22 \%$ from relapse and $4 \%$ from defaulter cases. This represents an increasing awareness of TB among people and a regular follow up from DOTS, which has significantly reduced defaulter cases from harbouring resistant strains, in contrary to previous studies from both India and other developing countries [18-20]. However, a significant number of failure and relapse cases from this study prompts more attention, since tubercular patients maybe spreading MDR strains in community. Also, failure cases were major risk among these patients, necessitating need for strengthening DOTS regimen regarding compliance and monitoring patients who may become drug resistant in future.

Few of limitations of the study were short duration of study since treatment for MDR TB usually exceeds more than one and half year and prognosis of these patients on a long run would show more insight into the disease. Side effect profile of drugs and characterization based on severity of disease were not performed. Since these patients are on multiple drugs, individual drug toxicity cannot be ascertained. However they are important to address to maintain the compliance for treatment. Disease severity, socioeconomic factors, malnutrition and compliance to treatment are important parameters in these MDR patients which would represent prognosis. Due to each of them acting as confounding factors, they were not described in the study.

\section{Conclusion}

The study shows the usefulness of risk factors such as presence of breathlessness, lower BMI, radiological features of cavitary lesion and reticulonodular pattern as screening tools in identifying individuals 
with MDR-TB. Also, HIV infection was risk factor for non-MDR-TB, but not for MDR cases. Pleural effusion in presentation was predominantly seen in non MDR cases. These parameters maybe considered for early suspicion for drug resistance in index presentation. This group will benefit from close monitoring and early drug susceptibility testing. Although these parameters may never replace drug susceptibility testing, this helps in identification of at risk patients for surveillance, confirmation of results and family screening in high risk groups.

\section{Conflicts of interest}

Authors declare no conflicts of interest.

\section{References}

[1] World Health Organization (WHO). Global Tuberculosis Report 2018. Geneva:WHO. 2018. Accessed from: https:// apps.who.int/iris/handle/10665/274453

[2] Raviglione MC, Smith IM. XDR tuberculosis-implications for global public health. N Engl J Med. 2007; 356(7):656659.

[3] Johnston JC, Shahidi NC, Sadatsafavi M, Fitzgerald JM. Treatment outcomes of multidrug-resistant tuberculosis: a systematic review and meta-analysis. PLoS One. 2009; 4(9):e6914.

[4] Anderson LF, Tamne S, Watson JP, Cohen T, Mitnick C, et al. Treatment outcome of multi-drug resistant tuberculosis in the United Kingdom: retrospective-prospective cohort study from 2004 to 2007. Euro Surveill. 2013; 18(40):20601.

[5] Diandé S, Sangaré L, Kouanda S, Dingtoumda BI, Mourfou A, et al. Risk factors for multidrug-resistant tuberculosis in four centers in Burkina Faso, West Africa. Microb Drug Resist Dis 2009; 15(3):217-221.

[6] Elmi OS, Hasan H, Abdullah S, Mat Jeab MZ, Zilfalil BA, et al. Treatment outcomes of patients with multidrug-resistant tuberculosis (MDR-TB) compared with non-MDR-TB infections in Peninsular Malaysia. Malays J Med Sci 2016; 23(4):17-25.

[7] Faustini A, Hall AJ, Perucci CA. Risk factors for multidrugresistant tuberculosis in Europe: a systematic review. Thorax 2006; 61:158-163.

[8] Wondemagegn M, Mekonnen D, Yimer M, Admassu A, Abera B. Risk factors for multidrug-resistant tuberculosis patients in amhara national regional state. Afr Health Sci 2015; 15(2):368-377.

[9] Chuchottaworn C, Thanachartwet V, Sangsayunh P, Than TZM, Sahassananda D, et al. Risk factors for multidrugresistant tuberculosis among patients with pulmonary tuberculosis at the central chest institute of Thailand. PLoS One 2015; 10(10):e0139986.

[10] Baya B, Achenbach CJ, Kone B, Toloba Y, Dabitao DK, et al. Clinical risk factors associated with multidrug-resistant tuberculosis (MDR-TB) in Mali. Int J Infect Dis. 2019; 81:149-155.

[11] Lukoye D, Adatu F, Musisi K, Kasule GW, Were W, et al. Antituberculosis drug resistance among new and previously treated sputum smear-positive tuberculosis patients in Uganda: results of the first national survey. PLoS One. 2013; 8(8):e70763.
[12] Mesfin YM, Hailemariam D, Biadglign S, Kibret KT. Association between HIV/AIDS and multi-drug resistance tuberculosis: a systematic review and meta-analysis. PLoS One 2014; 9(1):e82235.

[13] Cherkaoui I, Sabouni R, Ghali I, Kizub D, Billioux AC, et al. Treatment default amongst patients with tuberculosis in urban Morocco: Predicting and explaining default and postdefault sputum smear and drug susceptibility results. PLoS One. 2014; 9(4):e93574.

[14] Atif M, Bashir A, Ahmad N, Fatima RK, Saba S, et al. Predictors of unsuccessful interim treatment outcomes of multidrug resistant tuberculosis patients. BMC Infect Dis. 2017; 17(1):1-12.

[15] Mollel EW, Chilongola JO. Predictors for Mortality among multidrug-resistant tuberculosis patients in Tanzania. J Trop Med. 2017; 2017:9241238.

[16] Heuvel AVD, Smet H, Prat I, Sands A, Urassa W, et al. Laboratory evaluation of four HIV/syphilis rapid diagnostic tests. BMC Infect Dis. 2019 Jan 3; 19(1):1.

[17] Larsson G, Shenoy T, Ramasubramanian R, Balakumaran LK, Småstuen MC, et al. Routine diagnosis of intestinal tuberculosis and Crohn's disease in Southern India. World J Gastroenterol. 2014; 20(17):5017-5024.

[18] Charan J, Tank N, Reljic T, Singh S, Bhardwaj P, et al. Prevalence of multidrug resistance tuberculosis in adult patients in India: A systematic review and meta-analysis. J Family Med Prim Care. 2019; 8(10):3191-3201.

[19] Chatterjee S, Poonawala H,Jain Y. Drug-resistant tuberculosis: is India ready for the challenge?. BMJ Glob Health. 2018; 3(4): 000971.

[20] Goyal V, Kadam V, Narang P, Singh V. Prevalence of drugresistant pulmonary tuberculosis in India: Systematic review and meta-analysis. BMC Public Health. 2017; 17:817. 